\title{
3 Tesla Magnetic Resonance Imaging
}

National Cancer Institute

\section{Source}

National Cancer Institute. 3 Tesla Magnetic Resonance Imaging. NCI Thesaurus. Code C93013.

MRI that uses a magnet capable of producing a 3 telsa field strength. In theory, 3.0 T magnets have the capability to provide better image quality, with improved diagnostic performance. 